
capture enrichment

3 Megan S. Beaudry ${ }^{1, \#, *}$, Jincheng Wang ${ }^{1,2, \#, \ddagger \text {, Troy J. Kieran }{ }^{1} \text {, Jesse Thomas }}{ }^{1,3, \S}$, Natalia J. Bayona-

4 Vásquez $^{1,4, \delta}$, Bei Gao ${ }^{1}$, Alison Devault ${ }^{5}$, Brian Brunelle ${ }^{5}$, Kun Lu ${ }^{1, \text {, }}$, Jia-Sheng Wang ${ }^{1,2}$, Olin E.

5 Rhodes, Jr. ${ }^{3}$, Travis C. Glenn ${ }^{1,2,4}$,

$6{ }^{1}$ Department of Environmental Health Science, University of Georgia, Athens, GA 30602, USA

$7 \quad{ }^{2}$ Interdisciplinary Toxicology Program, University of Georgia, Athens, GA 30602, USA

$8 \quad{ }^{3}$ Savannah River Ecology Laboratory, University of Georgia, Aiken, SC 29808, USA

$9{ }^{4}$ Institute of Bioinformatics, University of Georgia, Athens, GA 30602, USA

$10{ }^{5}$ Daicel Arbor Biosciences, 5840 Interface Dr., Suite 101, Ann Arbor, MI 48103, USA

11 \#equal contributions (co-first authors)

12 tcurrent address: Department of Biochemistry and Microbiology, Rutgers University, New Brunswick, 13 NJ 08854, USA

14 \&current address: Center for Disease Control, Atlanta, GA 30329, USA

$15 \delta$ current address: Department of Biology, Oxford College of Emory University, 801 Emory Street, 16 Oxford, GA, 30054, USA

"current address: School of Marine Sciences, Nanjing University of Information Science and

18 Technology, Nanjing, 210044, China

'current address: Department of Environmental Sciences and Engineering, University of North Carolina, Chapel Hill, NC 27599, USA

$21 *$ Correspondence:

22 Megan Beaudry

$23 \quad$ Megan.Beaudry@uga.edu

24 Keywords: amplicon, microbial diversity, microbiome, mock communities, next generation 25 sequencing, shotgun libraries, target enrichment

\title{
26 Abstract
}

27 Environmental microbial diversity is often investigated from a molecular perspective using $16 \mathrm{~S}$

28 ribosomal RNA (rRNA) gene amplicons and shotgun metagenomics. While amplicon methods are

29 fast, low-cost, and have curated reference databases, they can suffer from amplification bias and are

30 limited in genomic scope. In contrast, shotgun metagenomic methods sample more genomic regions

31 with fewer sequence acquisition biases. However, shotgun metagenomic sequencing is much more

32 expensive (even with moderate sequencing depth) and computationally challenging. Here, we 
33 develop a set of 16S rRNA sequence capture baits that offer a potential middle ground with the

34 advantages from both approaches for investigating microbial communities. These baits cover the

35 diversity of all 16S rRNA sequences available in the Greengenes (v. 13.5) database, with no

36 sequence having $<80 \%$ sequence similarity to at least one bait for all segments of $16 \mathrm{~S}$. The use of

37 our baits provide comparable results to $16 \mathrm{~S}$ amplicon libraries and shotgun metagenomic libraries

38 when assigning taxonomic units from $16 \mathrm{~S}$ sequences within the metagenomic reads. We demonstrate

39 that $16 \mathrm{~S}$ rRNA capture baits can be used on a range of microbial samples (i.e., mock communities

40 and rodent fecal samples) to increase the proportion of 16S rRNA sequences (average $>400$-fold) and

41 decrease analysis time to obtain consistent community assessments. Furthermore, our study reveals

42 that bioinformatic methods used to analyze sequencing data may have a greater influence on

43 estimates of community composition than library preparation method used, likely in part to the extent

44 and curation of the reference databases considered.

\section{Introduction}

46 The study of microbes is critically important, as they have many essential roles in ecosystem

47 function, disease pathology, host physiology, and possibly assessing infectious disease outbreaks

48 (Dueker et al., 2018; Gallardo-Escárate et al., 2020). As microbial communities can often be highly

49 diverse and complex, it can be challenging to identify rare taxa in complex environmental samples

50 (e.g., soil, freshwater, etc.) with traditional and modern techniques (i.e., culturing, 16S amplicons, or

51 metagenomic shotgun libraries). Advances in sequencing technologies have transformed traditional

52 microbiology. Microbial communities that were previously considered indiscernible or unstudied,

53 can now be investigated at greater depths than ever before from many different environmental

54 systems (Gilmour et al., 2010; Kustin et al., 2019).

55 For decades, the 16S small subunit ribosomal RNA (rRNA) gene has been the gold standard marker

56 for microbial molecular taxonomic research (Woese and Fox, 1977; Meola et al., 2015), as this

57 highly conserved gene contains nine rapidly evolving hypervariable regions that aid in species

58 identification (Yuan et al., 2015). Amplicon sequencing, targeting the 16S rRNA, is a cost-effective

59 and high-throughput method used to study aquatic, terrestrial, food- and host-associated microbial

60 communities (Logares et al., 2014; Polka et al., 2015; Jiang et al., 2016; Jousselin et al., 2016;

61 Jouglin et al., 2019; Suenami et al., 2019; Ziegler et al., 2019). However, studies relying on 16S

62 rRNA amplicon sequencing have limitations and biases. Relevant biases in 16S rRNA amplicon

63 sequencing are associated with DNA extraction, amplification via PCR, sequencing, and sequence 
64 analysis (Kennedy et al., 2014; Knight et al., 2018). Specifically, PCR biases include primer bias

65 (Klindworth et al., 2013; Kelly et al., 2019) and varying GC content (Aird et al., 2011). Additional

66 limitations associated with amplicon sequencing include challenges in the taxonomic characterization

67 of microbial communities, as well as accuracy and availability of reference databases (Kennedy et al.,

68 2014; Poretsky et al., 2014; Ritari et al., 2015; Knight et al., 2018). Furthermore, the selection of the

69 hypervariable region used for the amplicon analysis (i.e., V1-V3; V3-V4; V4; etc) can lead to

70 differences in bacterial identification (Vetrovsky and Baldrian, 2013; Martinez-Porchas et al., 2016).

71 In more recent years, metagenomic shotgun sequencing has aimed to characterize taxonomic profiles

72 of unique clade-specific marker genes to provide a balanced view of community composition and

73 function (Neelakanta and Sultana, 2013; Knight et al., 2018). However, metagenomic sequencing has

74 its own limitations; genomic DNA may contain non-target DNA (e.g., human DNA), which can

75 affect downstream analysis (e.g., mis-assemblies of sequence contigs, spurious reads) thus leading to

76 inaccurate conclusions (Schmieder and Edwards, 2011; Gasc and Peyret, 2018). Also, metagenomic

77 libraries are more expensive, take longer to prepare, and are much more complex than amplicon

78 libraries, requiring more computational effort (Sekse et al., 2017). In particular, it is difficult to

79 identify low abundance genetic traits and rare taxa using metagenomic libraries, and extensive deep

80 sequencing is often required to do so (Lasa et al., 2019). In summary, shotgun sequencing is less

81 biased and yields data on many genomic regions, but the main tradeoffs are high costs of library

82 preparation, sequencing, and analysis.

83 Mock communities can be used to help establish ground truth in microbial diversity studies, in

84 particular when comparing different library preparation methods (Costea et al., 2017; Rausch et al.,

85 2019). Rausch et al., 2019 provided a comparison of 16S rRNA amplicon sequencing and

86 metagenomic sequencing, and revealed similar community makeup (i.e., abundance and taxa

87 diversity) of their shallow mock community regardless of library type. Conversely, other studies have

88 found key differences in abundance and taxa of mock communites attributed to wet-laboratory

89 methods (Costea et al., 2017; Rausch et al., 2019). However, some of these differences may be

90 attributed to varying bioinformatic tactics.

91 In terms of bioinformatic analyses, advantages and limitation of methods, reference databases, and

92 software have been vastly described for both 16S rRNA and metagenomic strategies (Truong et al.,

93 2015; Callahan et al., 2016a; Costea et al., 2017; Escobar-Zepeda et al., 2018; Rausch et al., 2019).

94 The variation among these can lead to a lack of sensitivity and specificity that may contribute to

95 wrong classifications and/or no classification at a specific taxonomic level, and erroneous abundance 
assignments (Escobar-Zepeda et al., 2018). In particular, it can be challenging to analyze environmental samples, as most reference databases are based on human commensals (Dueholm et al., 2020).

Both strategies (i.e., 16S rRNA amplicon and metagenomic shotgun libraries) present their own challenges and variations in analyses (Knight et al., 2018), but metagenomic shotgun libraries tend to perform at a higher sensitivity and specificity than 16S rRNA amplicon data (Escobar-Zepeda et al., 2018). For metagenomic data, programs like MetaPhlAn2 may be used to classify and estimate the relative abundance of microbial cells by mapping reads against marker sequences to classify the sequences at the sub-species to higher taxonomic levels (i.e., marker-gene approach) (Segata et al., 2012; Truong et al., 2015). Whereas $16 \mathrm{~S}$ rRNA amplicon data is commonly analyzed by inferring representative sequences using a variety of methods, some of which are influenced by fragment size and 16S region (Edgar, 2013; Callahan et al., 2016a; Callahan et al., 2016b). Furthermore, some methods used to assign operational taxonomic units may result in limited resolution at lower taxonomic levels (e.g., genus and species levels), as even organisms that share $98.75 \%$ sequences may be different species (Mysara et al., 2017). Reference databases for 16S rRNA are much more extensive than those for metagenomic analyses, which is key for superior analysis, particuraly in environmental samples (Escobar-Zepeda et al., 2018). However, variation in taxonomic classification and abundance has also been associated with the use of different reference databases (Jovel et al.,

114 2016; Rausch et al., 2019).

115 Hybridization capture (also known as sequence capture, target capture, or targeted sequence capture) 116 is an enrichment technique that uses a set of biotinylated DNA or RNA baits that are complementary 117 to DNA sequences of interest to increase the proportion of DNA fragments of interest within DNA 118 libraries, subsequently characterizing the DNA by massively parallel sequencing (Lasa et al., 2019).

119 Hybridization capture assays have been designed previously for the 16S rRNA gene, using 15-1,402 120 baits (Gasc and Peyret, 2018; Barrett et al., 2020). Additional hybridization capture bait sets have 121 been designed for a variety of microbial projects, such as sets of defined pathogens or particular 122 genes, including virulence genes for Vibrio spp. that infect oysters (Lasa et al., 2019), bifidobacterial 123 in the gut of mammals (Lugli et al., 2019), and antibiotic resistance genes (Guitor et al., 2019).

124 Importantly, unlike other culture independent techniques, hybridization capture provides greater 125 phylogenetic resolution and increased sensitivity, while requiring fewer sequencing reads (Lasa et al., 126 2019; Barrett et al., 2020). More specifically, 16S rRNA capture baits provide a cost-effective way to identify bacteria in diverse environmental samples and identify rare taxa. 
128 Here, we present a hybridization capture method (i.e., 16S-cap) to enrich metagenomic shotgun

129 libraries for DNA sequences of 16S rRNA genes. Our protocol improves on the existing methods by

130 including many more baits that better cover known sequence variation in 16S databases, taking

131 advantage of the extensive reference databases and ease of analyses of $16 \mathrm{~S}$ rRNA sequences for

132 taxonomic classification and decreasing bias introduced from primer affinity, while reducing

133 sequencing costs per sample compared to unenriched metagenomic libraries. For microbes, targeted

134 sequence capture techniques for 16S rRNA have shown more accurate representation of microbial

135 communities compared to traditional methods (i.e., 16S rRNA amplicons, shotgun libraries) (Gasc

136 and Peyret, 2018). We provide a comparison of traditional methods for assessing composition of

137 microbial communities (i.e., 16S rRNA amplicons and metagenomic shotgun libraries) with our 16S-

138 cap method to characterize in silico mock, in vitro mock, and real microbial communities from

139 genomic data.

\section{Materials \& Methods}

\subsection{Samples and DNA Extraction}

142 We used two commercial standard genomic DNA mock community collections to characterize 143 simple communities (HM-276D, BEI Resources, Manassas, VA; D6306, Zymo Research, Irvine,

144 CA). For complex communities, we used a subset of fecal samples from previous studies that 145 examined the impacts of environmental xenobiotic agents on the gut microbial communities of 146 rodent models (Gao et al., 2017; Wang et al., 2018). The first study examined carbamate insecticide 147 in male C57BL/6 mice (i.e., Mus musculus) (Gao et al., 2017), and the second examined green tea 148 polyphenols in female Sprague-Dawley rats (i.e., Rattus norvegicus) (Wang et al., 2018). DNA was 149 extracted using Qiagen Fast DNA Stool Mini Kit (QIAGEN, Valencia, CA, USA) or PowerSoil 150 DNA Isolation Kit (Mo Bio Laboratories, Carlsbad, CA, USA). Details on experimental design and 151 extractions are previously described (Gao et al., 2017; Wang et al., 2018).

\section{$152 \quad 2.2 \quad 16 S$ rRNA Amplicon Metabarcoding}

153 The primer pairs targeting the V3 and V4 16S regions (S-D- Bact-0341-b-S-17 and S-D-Bact-0785154 a-A-21 ) (Klindworth et al., 2013) were used for amplification of the 16S rRNA gene in rat fecal 155 samples and mock communities; and the primer pair targeting the V4 region (515-F and 806-R) 156 (Caporaso et al., 2012) was used on the mouse fecal samples. We created indexed fusion primers with TruSeq compatible sequencing oligos as previously described using the Adapterama I and 
158

159

160

161

162

163

164

165

166

167

168

169

170

171

172

173

174

175

176

177

178

179

180

181

182

183

184

185

186

Adapterama II systems (Glenn et al., 2019a; Glenn et al., 2019b) to generate amplicon libraries using two rounds of PCR (Method 5 of Table 3 from Glenn et al. 2019b). For the first PCR, we prepared individual $25 \mu \mathrm{L}$ PCR reactions for each sample using KAPA HiFi reagents (KAPA Biosystems, Wilmington, MA, USA). Each PCR reaction mix included $5 \mu \mathrm{L}$ 5x KAPA HiFi buffer, $0.75 \mu \mathrm{L} 10$ mM dNTPs, $0.5 \mu \mathrm{L}$ KAPA HiFi HotStart, $1.5 \mu \mathrm{L} 5 \mu \mathrm{M}$ forward indexed-fusion primer, $1.5 \mu \mathrm{L} 5 \mu \mathrm{M}$ reverse indexed-fusion primer, and $1 \mu \mathrm{L}$ of $20 \mathrm{ng} / \mu \mathrm{L}$ DNA. PCR conditions were as follows: initial denaturation at $95^{\circ} \mathrm{C}$ for $3 \mathrm{~min} ; 15-18$ cycles of $95^{\circ} \mathrm{C}$ for $20 \mathrm{sec}, 60^{\circ} \mathrm{C}$ for $30 \mathrm{sec}$, and $72^{\circ} \mathrm{C}$ for 30 sec; final extension at $72^{\circ} \mathrm{C}$ for $5 \mathrm{~min}$.

In preparation for the second PCR, we normalized individually indexed PCR products with a SequalPrep Normalization Plate Kit (Invitrogen, Carlsbad, CA, USA) according to manufacturer's protocols or by pooling them together based on agarose gel band brightness. These pools served as the template for a second limited cycle PCR. Each $25 \mu \mathrm{L}$ PCR reaction mix included: $5 \mu \mathrm{L} 5 \mathrm{x}$ KAPA HiFi buffer, $0.75 \mu \mathrm{L} 10 \mathrm{mM}$ dNTPs, $0.5 \mu \mathrm{L}$ KAPA HiFi HotStart, $2.5 \mu \mathrm{L}$ of $5 \mu \mathrm{M}$ forward iTru5 primer, $2.5 \mu \mathrm{L}$ of $5 \mu \mathrm{M}$ reverse iTru7 primer, and $5 \mu \mathrm{L}$ of product from the first PCR. The following were used as PCR conditions: initial denaturation at $95^{\circ} \mathrm{C}$ for $2 \mathrm{~min} ; 10$ cycles of $95^{\circ} \mathrm{C}$ for $20 \mathrm{sec}$, $60^{\circ} \mathrm{C}$ for $15 \mathrm{sec}$, and $72^{\circ} \mathrm{C}$ for $30 \mathrm{sec}$; final extension at $72^{\circ} \mathrm{C}$ for $5 \mathrm{~min}$. These PCR products were purified with Sera-Mag magnetic beads (Thermo-Scientific, Waltham, MA, USA). We quantified the final products with a Qubit 2.0 Fluorometer (Thermo-Scientific, Waltham, MA, USA) and pooled them in equal molar ratios for sequencing. Samples were sequenced using an Illumina MiSeq v2 600 cycle kit (Illumina, San Diego, CA, USA) at the Georgia Genomics and Bioinformatics Core (Athens, GA, USA).

\subsection{Metagenomic Libraries}

Extracted DNA was sheared on a Bioruptor UCD-300 (Diagenode, Denville, NJ, USA) to an average size of about $500 \mathrm{bp}$. We input $\sim 100 \mathrm{ng}$ of fragmented DNA into each reaction of a KAPA HyperPrep Kit (KAPA Biosystems, Wilmington, MA, USA) following manufacturer's protocol at half volume reaction size with 14 PCR cycles using iTru adaptors and indexed primers (Glenn et al., 2019b). Samples were sequenced on an Illumina HiSeq 3000 with PE150 reads (Oklahoma Medical Research Foundation, Oklahoma City, OK, USA).

\subsection{S rRNA Bait Design}


We used Prokka v1.11 with default settings, to annotate and extract all 16S rRNA sequences in GreenGenes v13.5 to ensure that only 16S rRNA regions were represented in the final bait set (Seemann, 2014). Stretches of up to 25 Ns were replaced with T bases to facilitate probe design across short unknown regions. We then used USEARCH v8.1 (Edgar, 2010) to sort by length (large to short) and cluster (query coverage 90\%, identity 90\%) sequences, retaining one centroid from each cluster. We then designed 120mer baits with flexible $\sim 50 \%$ overlap. These baits were then clustered using USEARCH (query coverage 75\%, identity 78\%), and one centroid per cluster retained.

\subsection{S rRNA Hybridization Capture Enrichments}

Metagenomic libraries were combined into $500 \mathrm{ng}$ pools of eight samples for rodents or two samples for mock communities. Target enrichments of each pool were performed using myBaits kit (Arbor Biosciences CAT \# 308616, Ann Arbor, MI, USA) using the designed 16S rRNA Capture Baits following manufacturer's protocol (v3.01) with a 24-hour $65^{\circ} \mathrm{C}$ hybridization. Following hybridization, we used Dynabeads M-280 Streptavidin magnetic beads (Life Technologies, Carlsbad, CA, US) for capturing and washing each biotinyalted bait library. We then performed a postHiFi HotStart reagents (KAPA Biosystems, Wilmington, MA, USA) using $98^{\circ} \mathrm{C}$ for 45 seconds, followed by $16-22$ cycles of $98^{\circ} \mathrm{C}$ for 20 seconds, $60^{\circ} \mathrm{C}$ for 30 seconds, and $72^{\circ} \mathrm{C}$ for 60 seconds, ending with a final extension of $72^{\circ} \mathrm{C}$ for five minutes. PCR products were cleaned 1:1 with SeraMag beads (Glenn et al., 2019a), quantified on Qubit and pooled in equimolar ratios for sequencing paired-end $150 \mathrm{bp}$ and $300 \mathrm{bp}$ reads on Illumina HiSeq 3000 (Oklahoma Medical Research

Foundation, Oklahoma City, OK, USA) and MiSeq (Georgia Genomics Bioinformatics Core, Athens, GA, USA), respectively.

\subsection{Simulating 16S rRNA Target Enrichment Data}

210 Three metagenomes (i.e., Lindgreen synthetic metagenome (Lindgreen et al., 2016); Zymo Mock 211 Community DS6306 genomes; and BEI Mock Community HM-276D) were used to simulate 16S rRNA capture data. In summary, a fasta file containing our 120mer bait set was mapped to each metagenome fasta file (Supplementary Data 1-3) using Burrows-Wheeler aligner (bwa) v.0.7.17 (Li and Durbin, 2009). Samtools v1.9 (Li et al., 2009) was used to convert the obtained sam file into a bam file. This mapping process is meant to simulate what would be an error- and bias-free 
218 first position, if possible. Here, we sought to simulate a hybridization of the bait to the core of an $219 \sim 500 \mathrm{bp}$ fragment while obtaining the flanking regions typically captured from use of biotinylated 220 baits.

221 The software ART 2016.06.05 (Huang et al., 2012) was then used to simulate $>200,000$ paired-end

$222150 \mathrm{bp}$ fastq reads from these extended reference sequences from each metagenome. These fastq files

223 were mapped to Greengenes 97\% similarity database v.13.8 using BBmap v. 38.50 (Bushnell, 2014).

224 For each metagenome, we recorded the number of paired reads mapped to Greengenes, number of

225 forward reads, number of reverse reads and percentage average total mapped, and compared these

226 results with those from real samples also mapped to the Greengenes database (see below) (Altschul et

227 al., 1990).

\subsection{Data Processing and Analysis}

229 After obtaining demultiplexed Illumina pair-end raw sequences, we used library specific pipelines to 230 process the data (Figure 1). For 16S rRNA amplicon libraries, primers were removed using cutadapt 231 v1.15 (Martin, 2011). Following this, DADA2 (v1.8) was used for quality trimming and filtering, de232 replication and sequence-variant inference, merging paired-end reads, construction of feature tables, 233 low relative abundance filtering of $0.5 \%$, removal of chimeras, and taxonomy assignment (Callahan 234 et al., 2016a). The taxonomy assignment was based on 97\% clustered OUT based on Greengenes 235 v13.8 database in the DADA2 pipeline.

236 [Insert Figure 1]

237 For $16 \mathrm{~S}$-cap libraries, the resulting quality filtered reads were mapped to the $97 \%$ clustered OTU 238 based on Greengenes v13.8 database using BBmap v37.78 (Bushnell, 2014). The resulting mapping 239 information was filtered, and a hit was recorded if both ends of paired read hit the same reference, or 240 only one end of the paired read hit a reference. A low relative abundance filter of $0.5 \%$ was applied.

241 Also, we assessed the presence of non-target reads in the quality-filtered dataset by 1) running 242 MetaPhlAn2 v2.7.8 (Segata et al., 2012; Truong et al., 2015), and 2) mapping to the rat and mice 243 genomes using Burrows-Wheeler aligner (bwa) v.0.7.17 (Li and Durbin, 2009).

244 For unenriched metagenomic libraries, Trimmomatic v0.36 (Bolger et al., 2014) was used for quality 245 trimming using a sliding window of three nucleotides with an average $\mathrm{Q}>20$, and minimum length 246 of 75 nucleotides. Reads that passed initial quality filtering (including both paired reads and orphan 247 reads) were fed to MetaPhlAn2 v2.7.14 for taxonomy assignment (Segata et al., 2012; Truong et al., 248 2015). A low relative abundance filter of $0.5 \%$ was applied. To further compare to the results from 
249 16S-cap analysis, we performed the same 16S mapping steps to the GreenGenes database as

250 described for 16S-cap libraries for the unenriched libraries.

251 Data was analyzed using R statistical software (R Development Core Team, 2010). Duncan's

252 multiple range test was used to compare abundance estimates between library types. Additionally, for

253 all samples, abundance estimates were used to construct a Bray-Curtis dissimiarilty matrix, which

254 was then used to generate a prinicle coordinate analysis (PCoA).

\section{$2553 \quad$ Results}

\section{$256 \quad 3.1 \quad$ 16S rRNA Capture Bait Design}

257 The 1,262,986 sequences comprising Greengenes v13.5 were annotated and 1,261,075 16S rRNA

258 sequences were retained. A total of 117 sequences containing consecutive runs of 25 or more

259 ambiguous bases (Ns) were removed. A total of 18,649 centroidal sequences were obtained from

260 USEARCH clustering. From these sequences, 413,480 120mer baits were designed. These baits were

261 then clustered using USEARCH, retaining one centroid per cluster, for a total of 37,745 baits.

\section{$262 \quad 3.2 \quad$ Sequencing Summary Statistics}

263 A summary of average sequence statistics for each sample and library preparation type is given in

264 Table 1. For the 16S rRNA amplicon data, the number of total raw read pairs per sample ranged from 49,828 for the Zymo mock community to 136,184 for the BEI mock community, with rodent fecal samples having intermediate depth. More reads $(\sim 77 \%)$ remained from the rodent fecal samples after

267 the denoising steps through the rigorous DADA2 pipelines versus the mock communities. Low percentages of high quality reads remained following filtering for both the BEI and Zymo mock communities (38.7\% and 48.8\% respectively). For the BEI mock community, initial index matching in $\mathrm{R} 2$ reads caused $\sim 30 \%$ loss of data (versus less than 5\% typically observed in other samples) and DADA2 quality trimming lost another $30 \%$ of data. For the Zymo mock community, the loss of

272 data was mainly due to chimeric filtering ( $\sim 30 \%$ of data loss).

273 For the unenriched libraries, the highest number of total raw read pairs ranged from 4,985,957 in the

274 Zymo mock community to 28,219,552 in the insecticide-treated mouse feces. The percentage of

275 reads retained after filtering was greater than $65 \%$ for all unenriched libraries. The average

276 percentage of reads mapped to GreenGenes ranged from $0.1 \%$ to $0.2 \%$ in the BEI and Zymo mock communities. 
278 For 16S-cap libraries, the PE150 reads had higher numbers of reads on average per sample type than

279 PE300 reads. The highest number of raw reads (i.e., 11,474,476) was obtained for the insecticide-

280 treated mouse feces with PE150 reads. The percentage of reads after filtering were greater than $70 \%$

281 for all 16S-cap libraries. The average percentage of mapped reads was greater than 50\% for all 16S-

282 cap libraries, with the highest percentage of mapping in the 16S-cap BEI mock community

283 sequenced with PE300 at 75.7\%. On average among all sample types, the proportion of on target

284 reads was increased 435-fold when compared to unenriched libraries (range 283 - 499 fold increase,

285 Suppplemental Table 2).

$286 \quad$ [Insert Table 1]

287

288

289

290

291

292

293

294

295

296

297

298

299

300

301

302

303

304

305

306

307

308

\subsection{S rRNA Target Enrichment Simulated Reads}

Summary information for simulated reads is given in Table 2. We observed a higher percentage of

total mapped reads in our simulated mock communities than for the real data from those communities (Table 2). For example, the real data from the Zymo mock community had an average total mapping of $78.15 \%$ to GreenGenes, compared to $91.43 \%$ from the simulated data. Similarly, the BEI mock community had an average total mapping of $78.62 \%$ for the real data, compared to $92.37 \%$ for the simulated data.

[Insert Table 2]

\subsection{Validation on Mock Community Samples}

We initially prepared amplicon libraries, unenriched metagenomic libraries, and enriched our metagenomic libraries using the target enrichment bait set (i.e., 16S-cap) we developed using two mock communities (Table 1). At the phylum level both samples appear to provide accurate identification of the microbes with good estimates of abundance, regardless of library type or data analysis method used (Figure 2). The 16S-cap samples and metagenomic samples generate one detection of a false positive phyla in the mock community samples (Figure 2). Additionally, in both the unenriched and 16S-cap libraries analyzed with a 16S mapping approach, Cyanobacteria was found in low abundance even though it was not expected to be present in the mock community. However, when analyzing the unenriched library using marker gene approach, Cyanobacteria was not found and instead Ascomycota was identified.

[Insert Figure 2]

At the genus level, 16S-cap and unenriched libraries reflect more accurate microbial community composition and abundance for most taxa (Figure 3). The 16S-cap and unenriched libraries with 16S 
mapping missed three genera: Escherichia, Listeria, and Bacillus for both mock community samples. However, after identifying presumably false positive genera with above $1 \%$ abundance in the samples analyzed with $16 \mathrm{~S}$ mapping software, three families with no genus identification, genera. In comparison, $16 \mathrm{~S}$ rRNA amplicon-based analysis identified nearly all genera in mock samples, however, its estimates of abundance for Actinomyces, Propionibacterium, Pseudomonas, and Rhodobacter all greatly deviate from the nominal compositions. The unenriched metagenomic libraries analyzed with a marker-gene approach were able to identify all 18 genera in the mock communities, however its estimate of Bacillus abundance in both mock communities deviate from the nominal composition (Figure 3).

319 [Insert Figure 3]

320 [Insert Figure 4]

321 In the BEI mock community libraries, relative abundance estimates in the 16S-cap libraries were 322 more accurate than the amplicon and unenriched libraries as measured by fold change being very 323 close to 1 (Figure 4). In the amplicon library, several genera (i.e., Pseudomonas, Actinomyces,

324 Propionilbacterium, and Rhodobacter) are beyond the 2-fold change of their nominal compositions.

325 In particular one genus, Rhodobacter, proved to be challenging for all three library preparation 326 methods for accurate estimation of relative abundance. Duncan's multiple range test revealed that

327 there were significant differences ( $p$-value $\leq 0.05)$ between the BEI mock community amplicon and 328 16S-cap libraries, whereas the unenriched libraries were not found to be significantly different than 329 the amplicon or 16S-cap libraries. For the Zymo mock community libraries, relative abundance 330 estimates in the 16S-cap libraries are more accurate than relative abundance estimates for the 331 amplicon library. However, Duncan's multiple range test did not detect a significant difference 332 between the three library types (i.e., amplicon, unenriched, and enriched) (Figure 4).

\section{$333 \quad 3.5 \quad$ Validation on Fecal Samples}

334 Principle coordinate analysis was performed on mock community samples and additional samples

335 from laboratory mice and rats to further validate the 16S-cap method. When Bray-Curtis was used to 336 construct the dissimilarity matrix, which considers abundance estimates, we found that regardless of 337 analyses at the level of family (Figure 5A, left) or genus (Figure 5B, right) similar themes emerged.

338 We observed that the mock community samples were similar to each other regardless of library type. 339 Conversely, in the mouse and rat samples, we found that the unenriched libraries analyzed with a 
marker-gene approach grouped together separately from amplicon, unenriched, and 16S-cap libraries,

341 all of which were analyzed with the 16S mapping approach.

342 [Insert Figure 5]

343 A comparison of Bray-Curtis distance was performed for rodent fecal samples at the level of family

344 and genus (Figure 6). This analysis revealed similar trends regardless of sample type or taxonomic

345 rank. The 16S-cap and unenriched libraries analyzed with 16S mapping approach showed to be the

346 most similar to each other, with a dissimilarity rate below 0.25 . Bray-Curtis dissimilarity was higher

347 when comparing the amplicon libraries to both 16S-cap and unenriched libraries. When comparing

348 the unenriched libraries analyzed with two different analysis strategies (i.e., mapping reads to

349 GreenGenes vs gene-marker approach), we observed the highest degree of dissimilarity at both the

350 family and genus levels with dissimilarity rates at approximately 0.75 . Post-hoc analysis revealed

351 that there were significant differences when comparing the unenriched and 16S-cap libraries to all

352 other library types, regardless of sample type or taxonomic rank (Figure 6).

353 [Insert Figure 6]

\section{Discussion}

355 Given the limitations of $16 \mathrm{~S}$ rRNA amplicon and shotgun metagenomic libraries outlined in the 356 introduction, we sought to provide an alternative method to identify microbial community 357 composition by creating a 16S rRNA hybridization capture assay (i.e., 16S-cap). Our study revealed 358 two important things: 1) our 16S-cap method is an efficient way to obtain sequences from the 359 complete 16S rRNA gene to accurately reflect microbial community composition and abundance and 360 2) bioinformatic analysis methods greatly influence community composition in environmental 361 samples, regardless of library type. In our study we observed that sequences from 16S-cap were not 362 significantly different than sequences from unenriched shotgun libraries when analyzed using similar 363 bioinformatic methods and databases. However, we did find that the 16S-cap assay requires far fewer 364 reads, thus allowing enriched libraries to be characterized on benchtop sequencers, including 365 Illumina MiSeq instruments, at reasonable cost while overcoming the previously mentioned 366 limitations with direct 16S rRNA approaches and metagenomic approaches. These limitations

367 include selection and drift bias in PCR during amplicon library preparation and the potential for non368 target DNA (e.g., human DNA) in metagenomic libraries, which can lead to errors in downstream 369 analyses. 
370 Enrichment for genes of interest is an important technique in characterizing complex environmental

371 samples. Previous studies have found other capture enrichment methods to increase the proportion of

372 on target reads from $\sim 0.1 \%$ in unenriched shotgun libraries to $\sim 60 \%$ in enriched libraries (Gasc and

373 Peyret, 2018). Similarly, we found $0.1-0.2 \%$ of unenriched libraries to map to the16S rRNA,

374 whereas $58-76 \%$ of the enriched reads mapped to the 16S rRNA (Table 1). On average we achieved a

375435 -fold increase in reads mapped to the 16S rRNA in our 16S-cap libraries compared to the

376 unenriched libraries (Supplementary Table 2). In silico simulations of 16S-cap revealed that under

377 ideal conditions, $88-92 \%$ mapping to the 16S rRNA from mock communities could be achieved.

378 Therefore, our 16S-cap enrichment process helps to achieve a very high percentage of on-target

379 reads, but not quite as high as theoretically possible.

380 Our 16S-cap method identified several species that were not expected in the theoretical targets of the 381 mock communities, which may be attributed to several factors. First, the lack of genus identification 382 may be due to the mapping methods or clustering level used in data analysis rather than the library 383 preparation method. Both the 16S-cap and unenriched libraries analyzed with a $16 \mathrm{~S}$ mapping method 384 failed to identify three genera Escherichia, Listeria, and some Bacillus in the mock communities.

385 However, there are three familes, Enterobacteriaceae, Listeriaceae, and Bacillaceae, in the false 386 positive genera with $>1 \%$ abundance that align with our missing genera. Thus, it appears that reads 387 for these three genera appear to be present, but are not being assigned appropriately at the genus 388 level. By assigning these unidentified genera as Escherichia, Listeria, and Bacillus respectively, the 389 16S-cap library is highly accurate in terms of taxonomic classification and abundance. Taxonomic 390 misassignement is a known problem with $16 \mathrm{~S}$ mapping bioinformatic methods, and new software is 391 in development (Schloss and Westcott, 2011; Pollock et al., 2018; Zinger et al., 2019; Djemiel et al., 392 2020). Additional work on the mapping and assignment processes used here, as well as comparisons 393 of newly developed and commonly used bioinformatic software is beyond the scope of this paper, but 394 warranted in future work. Other taxa identified that were not expected in the mock communities may 395 be due to reagent contamination or index hopping during sequencing. Several studies show that 396 contaminating DNA is common in laboratory reagents and DNA extraction kits (Salter et al., 2014;

397 Weiss et al., 2014; Kim et al., 2017; Eisenhofer et al., 2019; Zinter et al., 2019). Furthermore, studies 398 recommend sequencing negative controls consisting of 'blank' extractions and library preparations to 399 identify contamination by bacterial species (Salter et al., 2014; Knight et al., 2018). Conversely, false 400 positives of extremely low abundance (i.e., $0.1 \%$ or less) may be due to misassigned data that can 
401 occur during Illumina sequencing. This phenomena is often referred to as index hopping (van der

402 Valk et al., 2019).

403 We compared theoretical target values of the BEI resources and Zymo mock communities to all three 404 library types (i.e., amplicon, unenriched, and 16S-cap) (Figures 3, 4). We find that the 16S-cap 405 libraries are representative of the target abundance values of the mock communities (Figure 3). Post406 hoc analysis revealed that the 16S rRNA amplicon library and 16S-cap library made from the BEI 407 mock community were significantly different from each other ( $p$-value $\leq 0.05$ ) based on relative 408 abundance. A PCoA revealed that in the mouse and rat samples the unenriched libraries analyzed 409 with a marker-gene approach grouped together separately from 16S rRNA amplicon libraries and 410 16S-cap and unenriched libraries analyzed with taxonomic binning approach (Figure 5). Thus, 411 enrichment and amplicon sequencing result in similar library composition, as do 16S-cap and 412 unenriched libraries analyzed with a 16S taxonomic binning approach. This indicates that our 16S413 cap method may be less biased than $16 \mathrm{~S}$ amplification, but that analysis methods or the reference 414 database may greatly influence community composition results. Walsh et al. (2018) analyzed 415 different species classifiers using marker gene approaches and taxonomic binning, and found that the 416 results of the marker gene approach (i.e., MetaPhlAn2) were different from taxonomic binning 417 methods. Taxonomic binning methods are influenced by the size of the reference genome, whereas 418 marker gene approaches are not (Droge and McHardy, 2012; Balvociute and Huson, 2017; Walsh et 419 al., 2018). The use of hybridization capture baits may help alleviate some of these issues.

420 Other groups have designed a more limited bait set to hybridize all known 16S rRNA gene sequences 421 by focusing on highly conserved regions and incorporating ambiguities(Gasc and Peyret, 2018).

422 When validating their bait set on a mock community, they found that they detected 24 of 26 genera 423 tested, and that two less abundant species (i.e., Methanobrevibacter smithii and Methanococcus 424 aelocius at $0.00006 \%$ ) were missed. In addition, Cariou et al., (2018) tested hybridization capture 425 probes designed by Gasc \& Peyret (2018) on a previously characterized pea aphid and found their 426 enriched libraries to be representative of the bacterial population. There are some key differences 427 between the design of our baits set and Gasc \& Peyret (2018). Foremost, is the number of baits 428 included in the bait set. Our bait set included 37,745 120mer baits, whereas Gasc \& Peyret bait set 429 include 15 baits that are 28- to 50-mer. Using more baits with more sequence variation among the 430 baits helps to capture a greater range of diverse targets and thus generates more accurate abundance 431 estimates of the full range of community members. Having a more extended bait set, as ours, may 432 allow to overcome some of the previous challenges, demonstrated by the ability to detect all genera 
433 in our mock communities. These aspects are critical when studying environmental samples and

434 searching for rare taxa. In addition, the use of longer hybridization times or "double capture" (i.e.,

435 when captured product is captured again) can improve the percentage of on target reads and help

436 capture rare sequences. Future work to identify the optimal bait set(s) for various microbial

437 communities and research objectives should include a direct comparison of the Gasc \& Peyret (2018)

438 bait set verses our bait set.

439 Preparing 16S-cap libraries can most readily be accomplished by using an existing enrichment kit, 440 which ranges in cost from $\$ 1,500$ - \$5,200 depending on the number of reactions purchased. To 441 reduce reagent costs and hands-on time, we have successfully pooled multiple samples (see section 442 2.5), which is commonly done (Glenn and Faircloth, 2016). For example, pooling samples in groups 443 of eight reduces capture costs from $\$ 93.75$ per sample to $\$ 11.72$ per sample (Supplementary Table

444 3). Larger numbers of samples can be pooled to further reduce costs, but there are tradeoffs (see 445 Glenn \& Faircloth, 2016). Our baitset is commercially available from Arbor Biosciences in ready-to446 use kit format, and the bait sequences are freely available to the scientific community

447 (Supplementary Data 4). Thus, our baits can be modified and/or synthesized by any strategy any 448 researcher desires.

449 Sequencing 16S-cap libraries require less extensive sequencing than unenriched shotgun

450 metagenomic libraries, which reduces costs (Supplementary Tables 4, 5). For example, a 100-fold

451 16S-cap enrichment sequenced on an Illumina MiSeq Nano PE150 provides a cost-savings of 452 approximately $\$ 315$ compared to an unenriched metagenomic shotgun library requiring 1 million 453 reads (Supplementary Table 4). Indeed, 16S-cap makes it economically and logistically reasonable to 454 routinely screen for $16 \mathrm{~S}$ segments from enriched shotgun metagenomic libraries on Illumina MiSeqs. 455 16S-cap decreases costs when using a production scale Illumina sequencer (e.g., Illumina NovaSeq) 456 to less than $\$ 0.10$ per sample when achieving a 100-fold enrichment (Supplementary Table 5).

457 However, because production scale sequencers produce 400 - 2,500 million read pairs, to achieve 458 low cost for samples needing relatively few reads, each run requires huge numbers of samples or a 459 mixture of some samples needing large numbers of reads (i.e., a mixture of projects; see Glenn et al. 460 2019a). Due to the limited savings possible on production sequencing costs (Supplementary Table 4), 461 the savings in data transfer, storage, and compute time may be more significant than savings in 462 sequencing costs.

463 In summary, our data demonstrates that the 16S-cap assay and unenriched shotgun metagenomic 464 libraries produce very similar community profiles. Importantly, our 16S-cap library is produced from 
465 a metagenomic library, which eliminates primer (though not all PCR) biases. Additionally, our 16S466 cap assay provides a deeper community profile (i.e., more $16 \mathrm{~S}$ reads that can be queried to a 467 database) with far fewer reads than the unenriched shotgun metagenomic libraries. In environmental 468 samples, we routinely achieved $>400$-fold enrichment. Thus, expensive deep sequencing is 469 unnecessary for $16 \mathrm{~S}$-cap libraries because a few thousand reads provide the same number of $16 \mathrm{~S}$

470 rRNA sequences as millions of shotgun reads. By trading modest additional library preparation costs 471 for reduced sequencing costs (Supplementary Tables 3-5), 16S cap is economical and opens up the 472 possibility of adding deep taxonomic sampling to studies that are capturing other genes of interests 473 (e.g., antibiotic resistance genes (Guitor et al., 2019; Oladeinde et al., 2019; Thomas et al., 2020). In 474 comparison to amplicon libraries, the 16S-cap assay will be more expensive, however, it provides 475 superior microbial community resolution, increased accuracy of relative abundance, and greater 476 flexiblity in terms of sequencer and kit choice. We believe that our bait set is a valuable tool to 477 efficiently and accurately identify microbial community composition and would be well-suited to be 478 used in combination with other bait sets targeting different genes of interest (e.g., antimicrobial 479 resistance baits).

Figures

481 Figure 1. Overview of data analysis methods on the three library types (i.e., 16s amplicon, 16s 482 hybridization bait capture, and metagenomic libraries).

483 Figure 2. Relative abundance of bacterial phyla in mock community controls sequenced and 484 analyzed using different methods. Phyla that are not among the nominal composition of the 485 respective mock communities are plotted as black dots next to z_Others. The black dot in the 486 enriched and unenriched library analzyed with 16S mapping software the assigned phyla was 487 Cyanobacteria. In unenriched libraries analyzed with a marker gene approach, the assigned phyla was 488 Ascomycota. Colored vertical bar in each panel represents the nominal abundance of respective 489 phylum. X-axis is plotted in log-scale to show the low abundance phylum. Row panel strips labels 490 identify the mock communities; column panel strips labels identify library type (i.e., amplicon, 491 enriched 16S-cap, unriched metagenomic library) and analyzing strategy (i.e., denoising, 492 16Smapping, and marker gene).

493 Figure 3. Relative abundance of bacterial genera in mock community controls sequenced and 494 analyzed using different methods. Panel A is the BEI mock community. Panel B is the Zymo mock 495 community. Genera that are not among the nominal composition of the respective mock communities 
were plotted as black dots under z_Others. Colored vertical bar in each panel represents the nominal abundance of respective genus. X-axis plotted in log-scale to show the low abundance genus. Row panel strips labels identify the mock communities; column panel strips labels identify library type

499 (i.e., amplicon, enriched 16S-cap, unriched metagenomic library) and analyzing strategy (i.e.,

500 denoising, 16Smapping, and marker gene).

501 Figure 4. Fold change (i.e., upper or under) comparing the relative abundances of respective genera 502 in each library to its nominal abundance. Duncan's multiple range test was performed to compare 503 each library type for each mock community. Letters indicate whether significant differences were 504 detected.

505 Figure 5. PCoA plots were constructed using Bray-Curtis dissimilarity matrix at a family level 506 (panel A) and genus level (panel B). Each project is represented by a colored dot (i.e., orange = BEI 507 mock community, green $=$ mouse samples, blue $=$ rat samples, and purple $=$ Zymo mock 508 community). Each library type, sequencing read length and data analysis method is represented by a 509 different shape (i.e., circle $=$ amplicon library, square $=16 \mathrm{~S}$-cap enriched PE150 reads, diamond = 510 unenriched PE150 analyzed with 16S mapping and triangle = unenriched PE150 analyzed with 511 metagenome mapping). Numbers represent sample number.

512 Figure 6. A comparison of the Bray-Curtis distance metric was performed for each library type at a 513 genus level using box plots. Bray-Curtis distance is indicated on the y-axis. Library type is indicated 514 on the x-axis. Duncan's multiple range test was performed to compare each library type for each 515 mock community. Letters indicate whether significant differences were detected.

\section{Permission to reuse and Copyright}

517 Figures, tables, and images will be published under a Creative Commons CC-BY licence and 518 permission must be obtained for use of copyrighted material from other sources (including re519 published/adapted/modified/partial figures and images from the internet). It is the responsibility of 520 the authors to acquire the licenses, to follow any citation instructions requested by third-party rights 521 holders, and cover any supplementary charges. 
523 Table 1. A brief overview of the average summary statistics (i.e., number of samples, total raw read-pairs, average filtered/bar, average 524 mapped/filtered) for each sample type of each library type (i.e., 16S amplicon libraries, 16S-cap enriched, and unenriched).

\begin{tabular}{|c|c|c|c|c|c|c|c|}
\hline $\begin{array}{l}\text { Library } \\
\text { Type }\end{array}$ & $\begin{array}{l}\text { Read } \\
\text { Length }\end{array}$ & $\begin{array}{l}\text { Sample } \\
\text { Type }\end{array}$ & $\begin{array}{l}\text { N } \\
\text { Samples }\end{array}$ & $\begin{array}{l}\text { Total Raw } \\
\text { Read-Pairs }\end{array}$ & $\begin{array}{l}\text { Total } \\
\text { Filtered } \\
\text { Reads }\end{array}$ & $\begin{array}{l}\text { Average } \\
\text { Filtered/Raw } \\
(\text { Mean } \pm \text { SD })\end{array}$ & $\begin{array}{l}\text { Average } \\
\text { Mapped/Filtered } \\
(\text { Mean } \pm \text { SD })\end{array}$ \\
\hline $\begin{array}{l}\text { Amplicon- } \\
\text { 16S/V3V4 }\end{array}$ & PE300 & Rat feces & 5 & 318,561 & 247,781 & $(77.3 \pm 6.2) \%$ & $\mathrm{NA}$ \\
\hline $\begin{array}{l}\text { Amplicon- } \\
\text { 16S/V3V4 }\end{array}$ & PE300 & $\begin{array}{l}\text { BEI } \\
\text { Mock }\end{array}$ & 1 & 136,184 & 52,734 & $38.7 \%$ & NA \\
\hline $\begin{array}{l}\text { Amplicon- } \\
\text { 16S/V3V4 }\end{array}$ & PE300 & $\begin{array}{l}\text { Zymo } \\
\text { Mock }\end{array}$ & 1 & 49,828 & 24,301 & $48.8 \%$ & NA \\
\hline $\begin{array}{l}\text { Amplicon- } \\
\text { 16S/V4 }\end{array}$ & PE250 & $\begin{array}{l}\text { Mice } \\
\text { feces }\end{array}$ & 8 & 526,754 & 389,000 & $(77.6 \pm 7.1) \%$ & NA \\
\hline Enriched & PE150 & $\begin{array}{l}\text { Mice } \\
\text { feces }\end{array}$ & 8 & $8,321,081$ & $11,474,476$ & $(70.1 \pm 5.4) \%$ & $(59.1 \pm 0.8) \%$ \\
\hline Enriched & PE150 & Rat feces & 5 & $6,450,541$ & $9,470,428$ & $(72.9 \pm 2.1) \%$ & $(57.8 \pm 4.1) \%$ \\
\hline Enriched & PE150 & $\begin{array}{l}\text { BEI } \\
\text { Mock }\end{array}$ & 1 & $5,345,638$ & $8,203,396$ & $76.7 \%$ & $70.4 \%$ \\
\hline Enriched & PE150 & $\begin{array}{l}\text { Zymo } \\
\text { Mock }\end{array}$ & 1 & $3,359,376$ & $5,140,030$ & $76.5 \%$ & $70.1 \%$ \\
\hline Enriched & PE300 & $\begin{array}{l}\text { Mice } \\
\text { feces }\end{array}$ & 8 & $1,050,608$ & $1,573,122$ & $(75.1 \pm 3.2) \%$ & $(59.9 \pm 2.1) \%$ \\
\hline Enriched & PE300 & $\begin{array}{l}\text { BEI } \\
\text { Mock }\end{array}$ & 1 & 737,309 & $1,108,481$ & $75.2 \%$ & $75.7 \%$ \\
\hline Enriched & PE300 & $\begin{array}{l}\text { Zymo } \\
\text { Mock }\end{array}$ & 1 & 467,250 & 721,740 & $77.2 \%$ & $73.8 \%$ \\
\hline Unenriched & PE150 & $\begin{array}{l}\text { Mice } \\
\text { feces }\end{array}$ & 8 & $28,219,552$ & $37,894,050$ & $(68.6 \pm 6.4) \%$ & $0.1 \%$ \\
\hline Unenriched & PE150 & Rat feces & 5 & $16,266,683$ & $28,448,468$ & $(87.4 \pm 0.9) \%$ & $0.1 \%$ \\
\hline Unenriched & PE150 & $\begin{array}{l}\text { BEI } \\
\text { Mock }\end{array}$ & 1 & $6,263,379$ & $8,889,636$ & $71 \%$ & $0.2 \%$ \\
\hline Unenriched & PE150 & $\begin{array}{l}\text { Zymo } \\
\text { Mock }\end{array}$ & 1 & $4,985,957$ & $7,001,503$ & $70.2 \%$ & $0.2 \%$ \\
\hline
\end{tabular}


527 Table 2. Summary statistics for simulated data and real data from mock communities, libraries were enriched for $16 \mathrm{~S}$ using the $16 \mathrm{~S}$-cap 528 enrichment and sequenced on an Illumina MiSeq PE150 reads.

\begin{tabular}{|c|c|c|c|c|c|c|c|c|}
\hline $\begin{array}{l}\text { Sample } \\
\text { ID }\end{array}$ & $\begin{array}{l}\text { Library } \\
\text { Type }\end{array}$ & $\begin{array}{l}\text { Avg. No. of } \\
\text { (Simulated) } \\
\text { Reads }\end{array}$ & $\begin{array}{l}\text { No. of } \\
\text { Simulated } \\
\text { Reads }\end{array}$ & $\begin{array}{l}\text { Matched } \\
\text { Pairs }\end{array}$ & $\begin{array}{l}\text { Matched } \\
\text { Forward }\end{array}$ & $\begin{array}{l}\text { Matched } \\
\text { Reverse }\end{array}$ & $\begin{array}{l}\text { Total } \\
\text { Mapped }\end{array}$ & $\begin{array}{l}\text { Percent } \\
\text { of Avg. } \\
\text { Total } \\
\text { Mapped }\end{array}$ \\
\hline \multicolumn{9}{|c|}{ Simulated Data } \\
\hline $\begin{array}{l}\text { Zymo } \\
\text { Mock }\end{array}$ & $\begin{array}{l}\text { Enriched- } \\
\text { PE150 }\end{array}$ & 412,520 & 206,260 & 171708 & 190,964 & 186,216 & 377,180 & $91.43 \%$ \\
\hline BEI Mock & $\begin{array}{l}\text { Enriched- } \\
\text { PE150 }\end{array}$ & 415,472 & 207736 & 176547 & 193998 & 189,777 & 383,775 & $92.37 \%$ \\
\hline $\begin{array}{l}\text { Lindgreen } \\
\text { et al., } \\
2016 \\
\end{array}$ & $\begin{array}{l}\text { Enriched- } \\
\text { PE150 }\end{array}$ & 490,238 & 245119 & 188620 & 218911 & 213,918 & 432,829 & $88.29 \%$ \\
\hline \multicolumn{9}{|l|}{ Real Data } \\
\hline $\begin{array}{l}\text { Zymo } \\
\text { Mock }\end{array}$ & $\begin{array}{l}\text { Enriched- } \\
\text { PE150 }\end{array}$ & $3,904,480$ & $1,952,240$ & $1,314,654$ & $1,548,323$ & $1,503,225$ & $3,051,548$ & $78.15 \%$ \\
\hline BEI Mock & $\begin{array}{l}\text { Enriched- } \\
\text { PE150 }\end{array}$ & $6,260,110$ & $3,130,055$ & $2,127,656$ & $2,486,274$ & $2,435,425$ & 4,921,699 & $78.62 \%$ \\
\hline
\end{tabular}




\section{Conflict of Interest}

531 The EHS DNA lab provides oligonucleotide aliquots and library preparation services at cost,

532 including some oligonucleotides and services used in this manuscript (baddna.uga.edu). Brian

533 Brunelle and Alison Devault are employed by, and thereby have financial interest in, Daicel Arbor

534 Biosciences, who provided the in-solution capture reagents used in this work.

\section{Author Contributions}

536 TG conceived of the project. JW, JT, TK, BG, KL, and TG designed experiments. JW, TK, and BG 537 performed the experiments. $\mathrm{AD}$ and $\mathrm{BB}$ designed the baits. JW and NB analyzed the data. AD, BB, $538 \mathrm{KL}, \mathrm{OR}, \mathrm{JSW}$, and TG provided funding and resources. MB wrote the manuscript. JW, TK, NB 539 wrote sections of the manuscript. MB and JW produced figures and tables. All authors critically 540 reviewed, edited, and approve of this work.

\section{$541 \quad$ Funding}

542 Funding for this grant was provided by the Center for Disease Control contract 200-2018-02889

543 (75D30118C02889), US Department of Energy Cooperative Agreement number DE-FC09-

544 07SR22506, National Institute of Health (R01ES024950, P30ES010126, and P42ES031007) and

545 United States Agency for International Development via Peanut and Mycotoxin Innovation

546 Laboratory (ECG-A00-13-00001-00). Daicel Arbor Biosciences provided the customized in-solution 547 capture reagents used in this work.

\section{Acknowledgments}

549 We acknowledge the contributions of Marissa Howard, Amanda Sullivan, Allison Perry, and Laura 550 Rose.

\section{Disclaimer}

552 This report was prepared as an account of work sponsored by agencies of the United States

553 Government. Neither the United States Government, nor any agency thereof, nor any of their

554 employees makes any warranty, express or implied, or assumes any legal liability or responsibility

555 for the accuracy, completeness, or usefulness of any information, apparatus, product, or process

556 disclosed or represents that its use would not infringe privately owned rights. Reference herein to any 
specific commercial product, process, or service by trade name, trademark, manufacturer, or otherwise does not necessarily constitute or imply its endorsement, recommendation, or favoring by the United States Government or any agency thereof. The views and opinions of authors expressed herein do not necessarily state or reflect those of the United States Government or any agency thereof.

\section{Bibliography}

Aird, D., Ross, M.G., Wei-Sheng, C., Danielsson, M., Fennell, T., Russ, C., et al. (2011). Analzying and minimizing PCR amplification bias in Illumina sequencing libraries. Genome Biology 12, $1-14$.

Altschul, S.F., Gish, W., Miller, W., Myers, E., and Lipman, D.J. (1990). Basic Local Alignment Search Tool. Journal of Molecular Biology 215, 403-410.

Balvociute, M., and Huson, D.H. (2017). SILVA, RDP, Greengenes, NCBI and OTT - how do these taxonomies compare? BMC Genomics 18(Suppl 2), 114. doi: 10.1186/s12864-017-3501-4.

Barrett, S.R., Hoffman, N.G., Rosenthal, C., Bryan, A., Marshall, D.A., Lieberman, J., et al. (2020). Sensitive identification of bacterial DNA in clinical specimens by broad range 16S rRNA enrichment. J Clin Microbiol, 1-30. doi: 10.1128/JCM.01605-20.

Bolger, A.M., Lohse, M., and Usadel, B. (2014). Trimmomatic: a flexible trimmer for Illumina sequence data. Bioinformatics 30(15), 2114-2120. doi: 10.1093/bioinformatics/btu170.

Bushnell, B. (2014). BBMAP: A fast, accurate, splice-aware aligner. Lawrence Berkeley National Laboratory.

Callahan, B.J., McMurdie, P.J., Rosen, M.J., Han, A.W., Johnson, A.J., and Holmes, S.P. (2016a). DADA2: High-resolution sample inference from Illumina amplicon data. Nat Methods 13(7), 581-583. doi: 10.1038/nmeth.3869.

Callahan, B.J., McMurdie, P.J., Rosen, M.J., Han, A.W., Johnson, A.J.A., and Holmes, S.P. (2016b). DADA2: High-resolution sample inference from Illumina amplicon data. Nature Methods 13(7), 581-583. doi: 10.1038/nmeth.3869.

Caporaso, J.G., Lauber, C.L., Walters, W.A., Berg-Lyons, D., Huntley, J., Fierer, N., et al. (2012). Ultra-high-throughput microbial community analysis on the Illumina HiSeq and MiSeq platforms. ISME J 6(8), 1621-1624. doi: 10.1038/ismej.2012.8.

Costea, P.I., Zeller, G., Sunagawa, S., Pelletier, E., Alberti, A., Levenez, F., et al. (2017). Towards standards for human fecal sample processing in metagenomic studies. Nat Biotechnol 35(11), 1069-1076. doi: 10.1038/nbt.3960.

Djemiel, C., Dequiedt, S., Karimi, B., Cottin, A., Girier, T., El Djoudi, Y., et al. (2020). BIOCOMPIPE: a new user-friendly metabarcoding pipeline for the characterization of microbial diversity from 16S, 18S and 23S rRNA gene amplicons. BMC Bioinformatics 21(1), 492. doi: 10.1186/s12859-020-03829-3.

Droge, J., and McHardy, A.C. (2012). Taxonomic binning of metagenome samples generated by next-generation sequencing technologies. Brief Bioinform 13(6), 646-655. doi: 10.1093/bib/bbs031.

Dueholm, M.S., Andersen, K.S., McIlroy, S.J., Kristensen, J.M., Yashiro, E., Karst, S.M., et al. (2020). Generation of Comprehensive Ecosystem-Specific Reference Databases with SpeciesLevel Resolution by High-Throughput Full-Length 16S rRNA Gene Sequencing and Automated Taxonomy Assignment (AutoTax). mBio 11(5). doi: 10.1128/mBio.01557-20. 
602

603

604

605

606

607

608

609

610

611

612

613

614

615

616

617

618

619

620

621

622

623

624

625

626

627

628

629

630

631

632

633

634

635

636

637

638

639

640

641

642

643

644

645

646

647

648

649

650

Dueker, M.E., French, S., and O'Mullan, G.D. (2018). Comparison of Bacterial Diversity in Air and Water of a Major Urban Center. Front Microbiol 9, 2868. doi: 10.3389/fmicb.2018.02868.

Edgar, R.C. (2010). Search and clustering orders of magnitude faster than BLAST. Bioinformatics 26(19), 2460-2461. doi: 10.1093/bioinformatics/btq461.

Edgar, R.C. (2013). UPARSE: highly accurate OTU sequences from microbial amplicon reads. Nat Methods 10(10), 996-998. doi: 10.1038/nmeth.2604.

Eisenhofer, R., Minich, J.J., Marotz, C., Cooper, A., Knight, R., and Weyrich, L.S. (2019).

Contamination in Low Microbial Biomass Microbiome Studies: Issues and

Recommendations. Trends Microbiol 27(2), 105-117. doi: 10.1016/j.tim.2018.11.003.

Escobar-Zepeda, A., Godoy-Lozano, E.E., Raggi, L., Segovia, L., Merino, E., Gutierrez-Rios, R.M., et al. (2018). Analysis of sequencing strategies and tools for taxonomic annotation: Defining standards for progressive metagenomics. Sci Rep 8(1), 12034. doi: 10.1038/s41598-01830515-5.

Gallardo-Escárate, C., Valenzuela-Muñoz, V., Núñez-Acuña, G., Valenzuela-Miranda, D., Castellón, F., Benavente-Cartes, B., et al. (2020). The wastewater microbiome: a novel insight for COVID-19 surveillance. Research Square, 1-20. doi: 10.21203/rs.3.rs-62651/v1.

Gao, B., Bian, X., Mahbub, R., and Lu, K. (2017). Sex-Specific Effects of Organophosphate Diazinon on the Gut Microbiome and Its Metabolic Functions. Environ Health Perspect 125(2), 198-206. doi: 10.1289/EHP202.

Gasc, C., and Peyret, P. (2018). Hybridization capture reveals microbial diversity missed using current profiling methods. Microbiome 6(1), 61. doi: 10.1186/s40168-018-0442-3.

Gilmour, M.W., Graham, M., Van Domselaar, G., Tyler, S., Kent, H., Trout-Yakel, K.M., et al. (2010). High-throughput genome sequencing of two Listeria monocytogenes clinical isolates during a large foodborne outbreak. BMC Genomics 11, 120. doi: 10.1186/1471-2164-11-120.

Glenn, T.C., and Faircloth, B.C. (2016). Capturing Darwin's dream. Mol Ecol Resour 16(5), 10511058. doi: 10.1111/1755-0998.12574.

Glenn, T.C., Nilsen, R.A., Kieran, T.J., Sanders, J.G., Bayona-Vasquez, N.J., Finger, J.W., et al. (2019a). Adapterama I: universal stubs and primers for 384 unique dual-indexed or 147,456 combinatorially-indexed Illumina libraries (iTru \& iNext). PeerJ 7, e7755. doi: 10.7717/peerj.7755.

Glenn, T.C., Pierson, T.W., Bayona-Vasquez, N.J., Kieran, T.J., Hoffberg, S.L., Thomas Iv, J.C., et al. (2019b). Adapterama II: universal amplicon sequencing on Illumina platforms (TaggiMatrix). PeerJ 7, e7786. doi: 10.7717/peerj.7786.

Guitor, A.K., Raphenya, A.R., Klunk, J., Kuch, M., Alcock, B., Surette, M.G., et al. (2019). Capturing the Resistome: a Targeted Capture Method To Reveal Antibiotic Resistance Determinants in Metagenomes. Antimicrob Agents Chemother 64(1), 1-37. doi: 10.1128/AAC.01324-19.

Huang, W., Li, L., Myers, J.R., and Marth, G.T. (2012). ART: a next-generation sequencing read simulator. Bioinformatics 28(4), 593-594. doi: 10.1093/bioinformatics/btr708.

Jiang, Y., Xiong, X., Danska, J., and Parkinson, J. (2016). Metatranscriptomic analysis of diverse microbial communities reveals core metabolic pathways and microbiome-specific functionality. Microbiome 4, 2. doi: 10.1186/s40168-015-0146-x.

Jouglin, M., Blanc, B., de la Cotte, N., Bastian, S., Ortiz, K., and Malandrin, L. (2019). First detection and molecular identification of the zoonotic Anaplasma capra in deer in France. PLoS One 14(7), e0219184. doi: 10.1371/journal.pone.0219184.

Jousselin, E., Clamens, A.L., Galan, M., Bernard, M., Maman, S., Gschloessl, B., et al. (2016). Assessment of a 16S rRNA amplicon Illumina sequencing procedure for studying the microbiome of a symbiont-rich aphid genus. Mol Ecol Resour 16(3), 628-640. doi: 10.1111/1755-0998.12478.

This is a provisional file, not the final typeset article 
651

652

653

654

655

656

657

658

659

660

661

662

663

664

665

666

667

668

669

670

671

672

673

674

675

676

677

678

679

680

681

682

683

684

685

686

687

688

689

690

691

692

693

694

695

696

697

Jovel, J., Patterson, J., Wang, W., Hotte, N., O'Keefe, S., Mitchel, T., et al. (2016). Characterization of the Gut Microbiome Using 16S or Shotgun Metagenomics. Front Microbiol 7, 459. doi: 10.3389/fmicb.2016.00459.

Kelly, R.P., Shelton, A.O., and Gallego, R. (2019). Understanding PCR Processes to Draw Meaningful Conclusions from Environmental DNA Studies. Sci Rep 9(1), 12133. doi: 10.1038/s41598-019-48546-x.

Kennedy, K., Hall, M.W., Lynch, M.D., Moreno-Hagelsieb, G., and Neufeld, J.D. (2014). Evaluating bias of illumina-based bacterial 16S rRNA gene profiles. Appl Environ Microbiol 80(18), 5717-5722. doi: 10.1128/AEM.01451-14.

Kim, D., Hofstaedter, C.E., Zhao, C., Mattei, L., Tanes, C., Clarke, E., et al. (2017). Optimizing methods and dodging pitfalls in microbiome research. Microbiome 5(1), 52. doi: 10.1186/s40168-017-0267-5.

Klindworth, A., Pruesse, E., Schweer, T., Peplies, J., Quast, C., Horn, M., et al. (2013). Evaluation of general 16S ribosomal RNA gene PCR primers for classical and next-generation sequencingbased diversity studies. Nucleic Acids Res 41(1), e1. doi: 10.1093/nar/gks808.

Knight, R., Vrbanac, A., Taylor, B.C., Aksenov, A., Callewaert, C., Debelius, J., et al. (2018). Best practices for analysing microbiomes. Nat Rev Microbiol 16(7), 410-422. doi: 10.1038/s41579-018-0029-9.

Kustin, T., Ling, G., Sharabi, S., Ram, D., Friedman, N., Zuckerman, N., et al. (2019). A method to identify respiratory virus infections in clinical samples using next-generation sequencing. Sci Rep 9(1), 2606. doi: 10.1038/s41598-018-37483-w.

Lasa, A., di Cesare, A., Tassistro, G., Borello, A., Gualdi, S., Furones, D., et al. (2019). Dynamics of the Pacific oyster pathobiota during mortality episodes in Europe assessed by 16S rRNA gene profiling and a new target enrichment next-generation sequencing strategy. Environ Microbiol 21(12), 4548-4562. doi: 10.1111/1462-2920.14750.

Li, H., and Durbin, R. (2009). Fast and accurate short read alignment with Burrows-Wheeler transform. Bioinformatics 25(14), 1754-1760. doi: 10.1093/bioinformatics/btp324.

Li, R., Yu, C., Li, Y., Lam, T.W., Yiu, S.M., Kristiansen, K., et al. (2009). SOAP2: an improved ultrafast tool for short read alignment. Bioinformatics 25(15), 1966-1967. doi: 10.1093/bioinformatics/btp336.

Lindgreen, S., Adair, K.L., and Gardner, P.P. (2016). An evaluation of the accuracy and speed of metagenome analysis tools. Sci Rep 6, 19233. doi: 10.1038/srep19233.

Logares, R., Sunagawa, S., Salazar, G., Cornejo-Castillo, F.M., Ferrera, I., Sarmento, H., et al. (2014). Metagenomic 16S rDNA Illumina tags are a powerful alternative to amplicon sequencing to explore diversity and structure of microbial communities. Environ Microbiol 16(9), 2659-2671. doi: 10.1111/1462-2920.12250.

Lugli, G.A., Duranti, S., Milani, C., Mancabelli, L., Turroni, F., Sinderen, D.V., et al. (2019). Uncovering Bifidobacteria via Targeted Sequencing of the Mammalian Gut Microbiota. Microorganisms 7(11), 1-11. doi: 10.3390/microorganisms 7110535.

Martin, M. (2011). Cutadapt removes adapter sequences from high-throughput sequencing reads. EMBnet 17(1), 10-12.

Martinez-Porchas, M., Villalpando-Canchola, E., and Vargas-Albores, F. (2016). Significant loss of sensitivity and specificity in the taxonomic classification occurs when short 16S rRNA gene sequences are used. Heliyon 2(9), e00170. doi: 10.1016/j.heliyon.2016.e00170.

Meola, M., Lazzaro, A., and Zeyer, J. (2015). Bacterial Composition and Survival on Sahara Dust Particles Transported to the European Alps. Front Microbiol 6, 1454. doi:

10.3389/fmicb.2015.01454. 
698

699

700

701

702

703

704

705

706

707

708

709

710

711

712

713

714

715

716

717

718

719

720

721

722

723

724

725

726

727

728

729

730

731

732

733

734

735

736

737

738

739

740

741

742

743

744

745

Mysara, M., Vandamme, P., Props, R., Kerckhof, F.M., Leys, N., Boon, N., et al. (2017).

Reconciliation between operational taxonomic units and species boundaries. FEMS Microbiol Ecol 93(4), 1-12. doi: 10.1093/femsec/fix029.

Neelakanta, G., and Sultana, H. (2013). The use of metagenomic approaches to analyze changes in microbial communities. Microbiol Insights 6, 37-48. doi: 10.4137/MBI.S10819.

Oladeinde, A., Cook, K., Lakin, S., Woyda, R., Abdo, Z., Looft, T., et al. (2019). Horizontal Gene Transfer and Acquired Antibiotic Resistance in Salmonella enterica Serovar Heidelberg following In Vitro Incubation in Broiler Ceca. Applied and Environmental Microbiology 85(22), e01903-01919.

Polka, J., Rebecchi, A., Pisacane, V., Morelli, L., and Puglisi, E. (2015). Bacterial diversity in typical Italian salami at different ripening stages as revealed by high-throughput sequencing of $16 \mathrm{~S}$ rRNA amplicons. Food Microbiol 46, 342-356. doi: 10.1016/j.fm.2014.08.023.

Pollock, J., Glendinning, L., Wisedchanwet, T., and Watson, M. (2018). The Madness of Microbiome: Attempting To Find Consensus "Best Practice" for 16S Microbiome Studies. Appl Environ Microbiol 84(7). doi: 10.1128/AEM.02627-17.

Poretsky, R., Rodriguez, R.L., Luo, C., Tsementzi, D., and Konstantinidis, K.T. (2014). Strengths and limitations of $16 \mathrm{~S}$ rRNA gene amplicon sequencing in revealing temporal microbial community dynamics. PLoS One 9(4), e93827. doi: 10.1371/journal.pone.0093827.

R Development Core Team (2010). (Vienna, Astria: R Foundation for Statistical Computing).

Rausch, P., Ruhlemann, M., Hermes, B.M., Doms, S., Dagan, T., Dierking, K., et al. (2019). Comparative analysis of amplicon and metagenomic sequencing methods reveals key features in the evolution of animal metaorganisms. Microbiome 7(1), 133. doi: 10.1186/s40168-0190743-1.

Ritari, J., Salojarvi, J., Lahti, L., and de Vos, W.M. (2015). Improved taxonomic assignment of human intestinal $16 \mathrm{~S}$ rRNA sequences by a dedicated reference database. BMC Genomics 16, 1056. doi: 10.1186/s12864-015-2265-y.

Salter, S.J., Cox, M.J., Turek, E.M., Calus, S.T., Cookson, W.O., Moffatt, M.F., et al. (2014). Reagent and laboratory contamination can critically impact sequence-based microbiome analyses. BM Biology 12(87), 1-12.

Schloss, P.D., and Westcott, S.L. (2011). Assessing and improving methods used in operational taxonomic unit-based approaches for 16S rRNA gene sequence analysis. Appl Environ Microbiol 77(10), 3219-3226. doi: 10.1128/AEM.02810-10.

Schmieder, R., and Edwards, R. (2011). Fast identification and removal of sequence contamination from genomic and metagenomic datasets. PLoS One 6(3), e17288. doi: 10.1371/journal.pone.0017288.

Seemann, T. (2014). Prokka: rapid prokaryotic genome annotation. Bioinformatics 30(14), 20682069. doi: 10.1093/bioinformatics/btu153.

Segata, N., Waldron, L., Ballarini, A., Narasimhan, V., Jousson, O., and Huttenhower, C. (2012). Metagenomic microbial community profiling using unique clade-specific marker genes. Nat Methods 9(8), 811-814. doi: 10.1038/nmeth.2066.

Sekse, C., Holst-Jensen, A., Dobrindt, U., Johannessen, G.S., Li, W., Spilsberg, B., et al. (2017). High Throughput Sequencing for Detection of Foodborne Pathogens. Front Microbiol 8, 2029. doi: 10.3389/fmicb.2017.02029.

Suenami, S., Konishi Nobu, M., and Miyazaki, R. (2019). Community analysis of gut microbiota in hornets, the largest eusocial wasps, Vespa mandarinia and V. simillima. Sci Rep 9(1), 9830. doi: 10.1038/s41598-019-46388-1.

Thomas, J.C.t., Oladeinde, A., Kieran, T.J., Finger, J.W., Jr., Bayona-Vasquez, N.J., Cartee, J.C., et al. (2020). Co-occurrence of antibiotic, biocide, and heavy metal resistance genes in bacteria

This is a provisional file, not the final typeset article 

Biotechnol 13(4), 1179-1200. doi: 10.1111/1751-7915.13578.

Truong, D.T., Franzosa, E.A., Tickle, T.L., Scholz, M., Weingart, G., Pasolli, E., et al. (2015). MetaPhlAn2 for enhanced metagenomic taxonomic profiling. Nat Methods 12(10), 902-903. doi: $10.1038 /$ nmeth.3589.

van der Valk, T., Vezzi, F., Ormestad, M., Dalen, L., and Guschanski, K. (2019). Index hopping on the Illumina HiseqX platform and its consequences for ancient DNA studies. Mol Ecol Resour, 1171-1181. doi: 10.1111/1755-0998.13009.

Vetrovsky, T., and Baldrian, P. (2013). The variability of the 16S rRNA gene in bacterial genomes and its consequences for bacterial community analyses. PLoS One 8(2), e57923. doi: 10.1371/journal.pone.0057923.

Walsh, A.M., Crispie, F., O'Sullivan, O., Finnegan, L., Claesson, M.J., and Cotter, P.D. (2018). Species classifier choice is a key consideration when analysing low-complexity food microbiome data. Microbiome 6(1), 50. doi: 10.1186/s40168-018-0437-0.

Wang, J., Tang, L., Zhou, H., Zhou, J., Glenn, T.C., Shen, C.L., et al. (2018). Long-term treatment with green tea polyphenols modifies the gut microbiome of female sprague-dawley rats. $J$ Nutr Biochem 56, 55-64. doi: 10.1016/j.jnutbio.2018.01.005.

Weiss, S., Amir, A., Hyde, E.R., Metcalf, J.L., Song, S.J., and Knight, R. (2014). Tracking down the sources of experimental contamination in microbiome studies. Genome Biology 15, 1-3.

Woese, C.R., and Fox, G.E. (1977). Phylogenetic structure of the prokaryotic domain: the primary kingdoms. Proc Natl Acad Sci US A 74(11), 5088-5090. doi: 10.1073/pnas.74.11.5088.

Yuan, C., Lei, J., Cole, J., and Sun, Y. (2015). Reconstructing 16S rRNA genes in metagenomic data. Bioinformatics 31(12), i35-i43. doi: 10.1093/bioinformatics/btv231.

Ziegler, M., Grupstra, C.G.B., Barreto, M.M., Eaton, M., BaOmar, J., Zubier, K., et al. (2019). Coral bacterial community structure responds to environmental change in a host-specific manner. Nat Commun 10(1), 3092. doi: 10.1038/s41467-019-10969-5.

Zinger, L., Bonin, A., Alsos, I.G., Balint, M., Bik, H., Boyer, F., et al. (2019). DNA metabarcodingNeed for robust experimental designs to draw sound ecological conclusions. Mol Ecol 28(8), 1857-1862. doi: 10.1111/mec.15060.

Zinter, M.S., Mayday, M.Y., Ryckman, K.K., Jelliffe-Pawlowski, L.L., and DeRisi, J.L. (2019). Towards precision quantification of contamination in metagenomic sequencing experiments. Microbiome 7(1), 62. doi: 10.1186/s40168-019-0678-6.

\section{Data Availability}

The datasets generated in this study can be found in the authors dropbox 

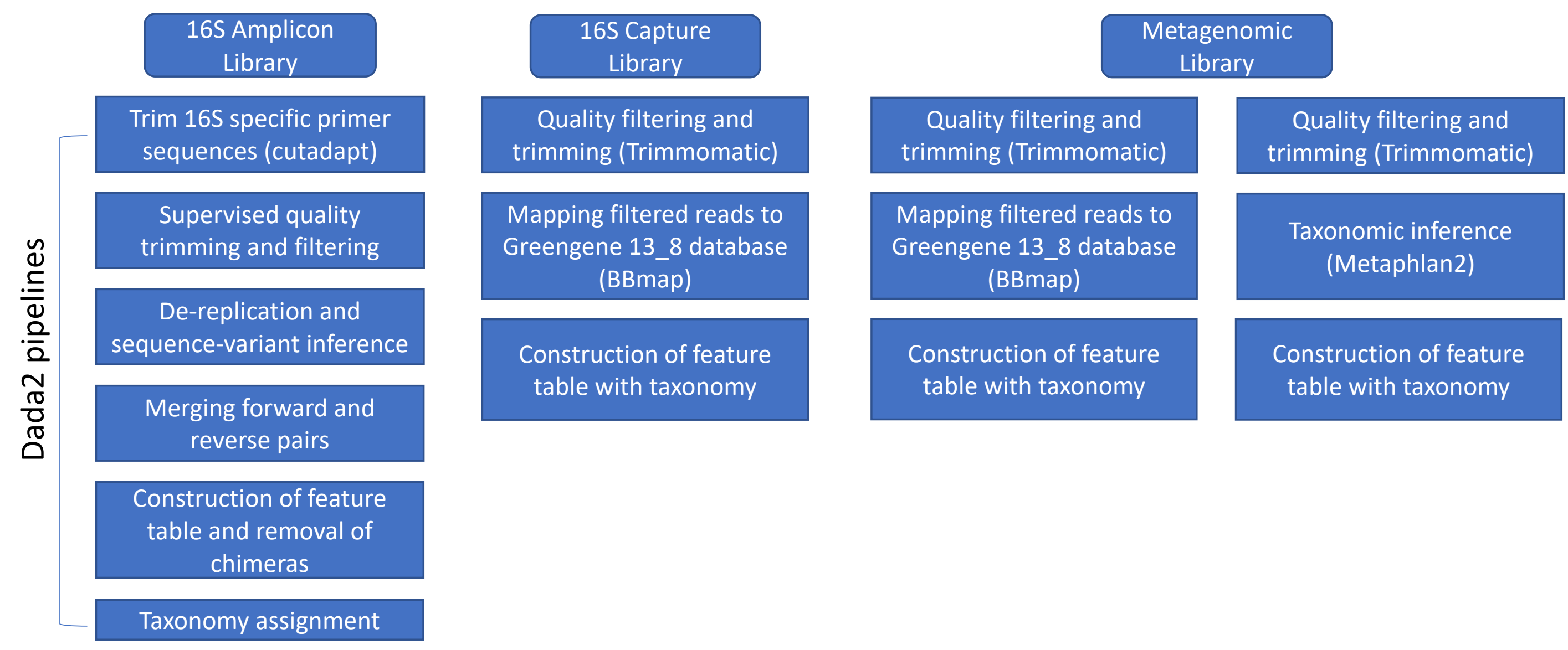


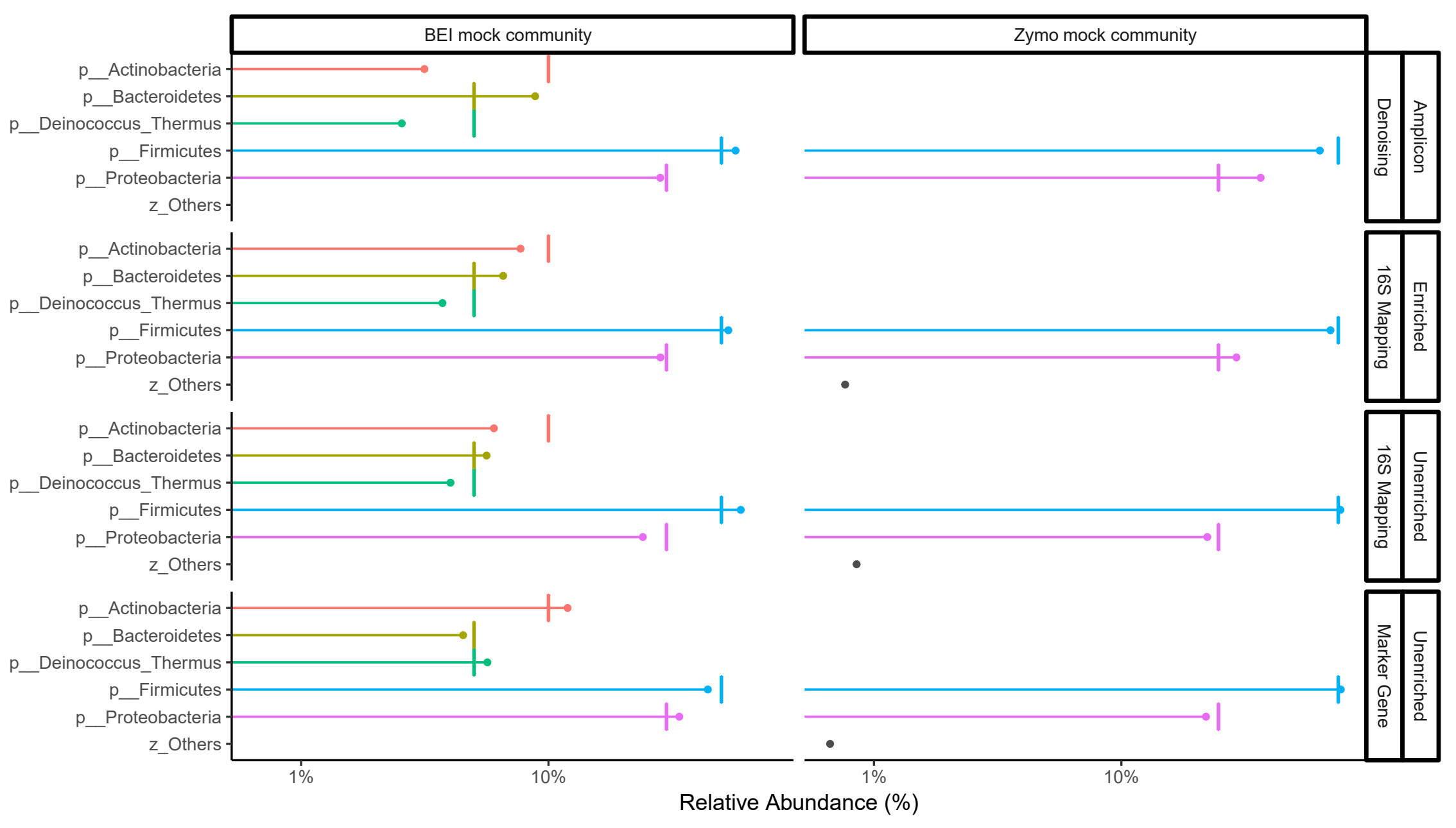


(A)

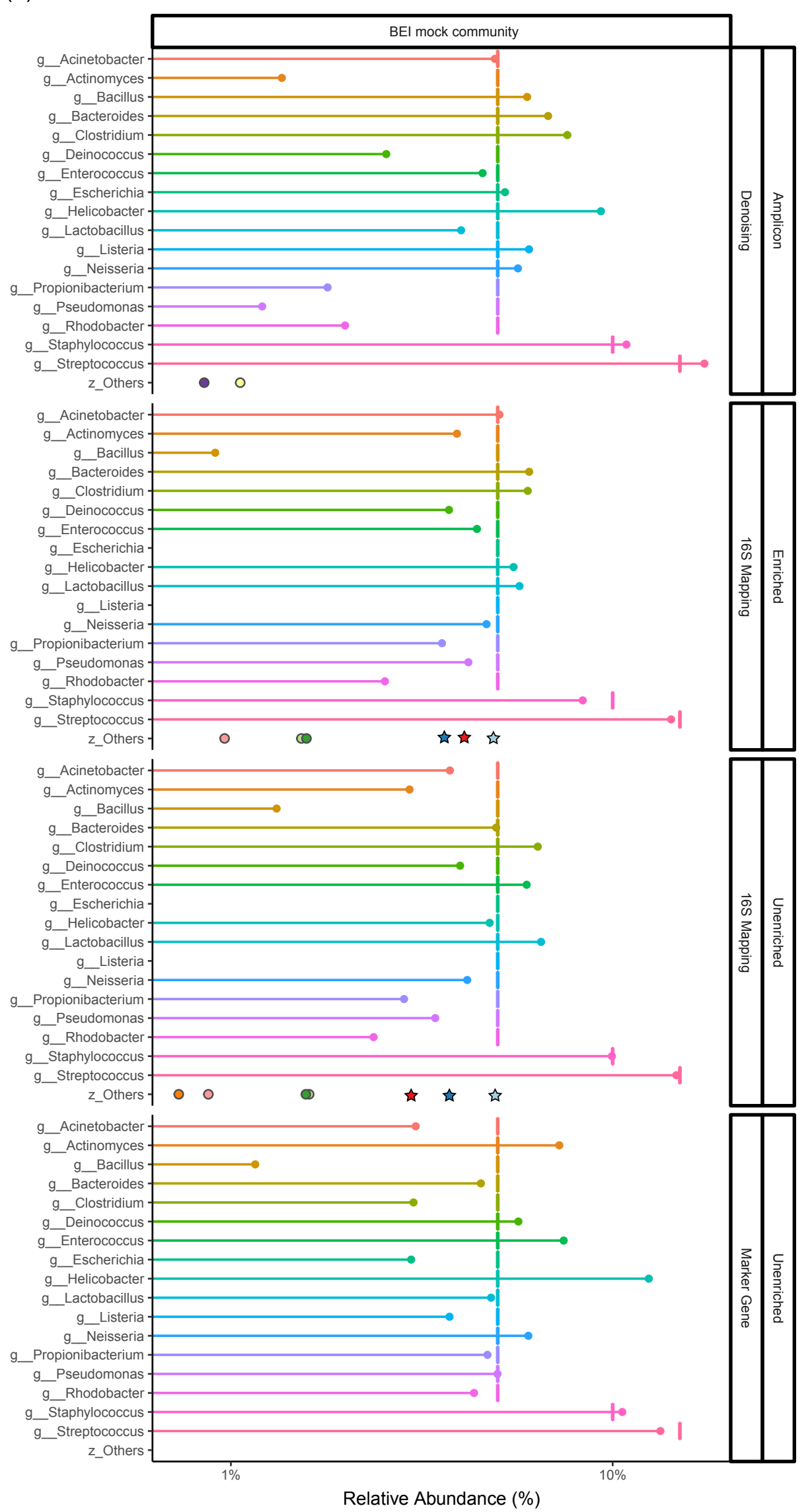

(B)

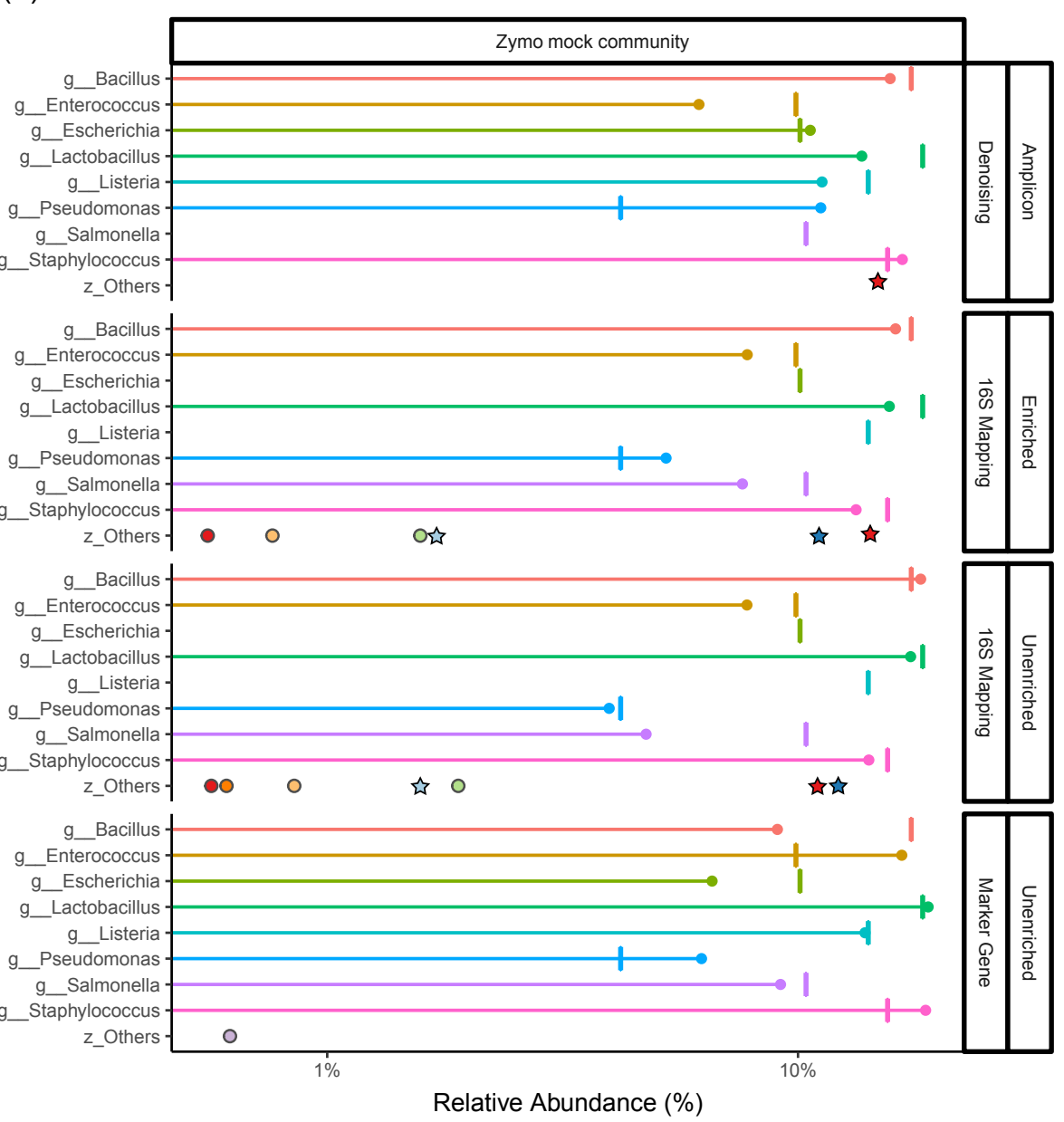

Family

\& f_Bacillaceae

$f$ Enterobacteriaceae

$f$ Listeriaceae

- f_Carnobacteriaceae

- f Clostridiaceae

- f Acaryochloridaceae

- f_Neisseriaceae

- f Planococcaceae

- f_Prevotellaceae

o f_S24-7

- f Saccharomycetaceae 
(A) Family Level PCoA

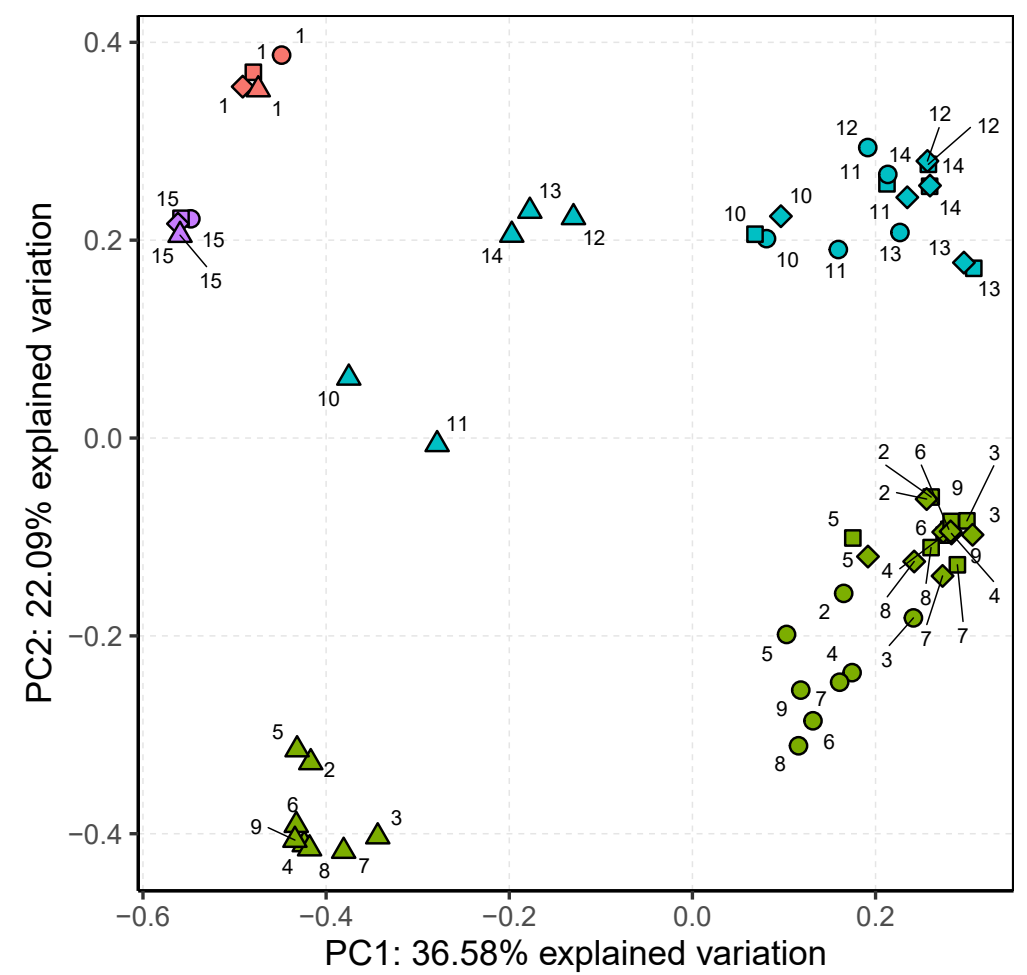

Projects
O BEI.mock
O Mouse
O Rat
○ Zymo.mock

(B) Genus Level PCoA

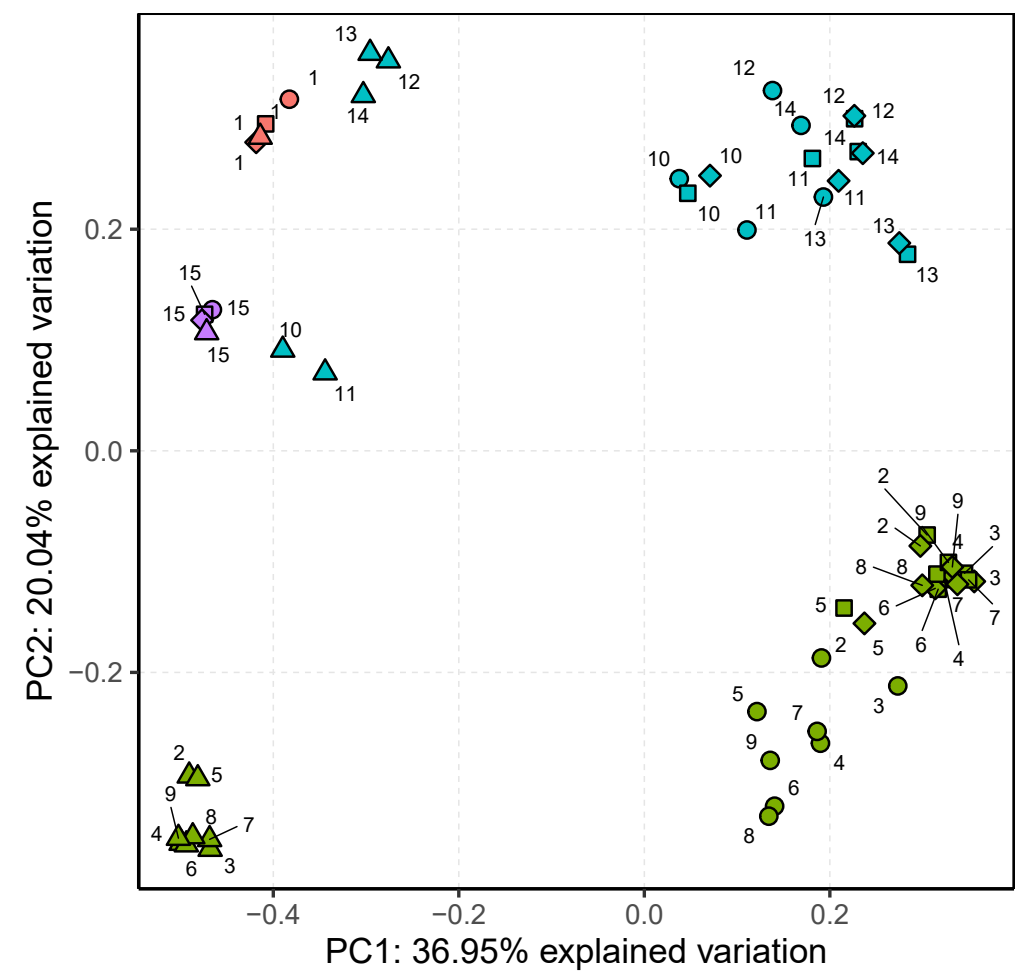

Library
O Amplicon_Denosing
Enriched_16SMapping
$\diamond$ Unenriched_16SMapping
$\triangle$ Unenriched_Marker 


\section{Comparison of bray-curtis distance}

Based on Genus level composition

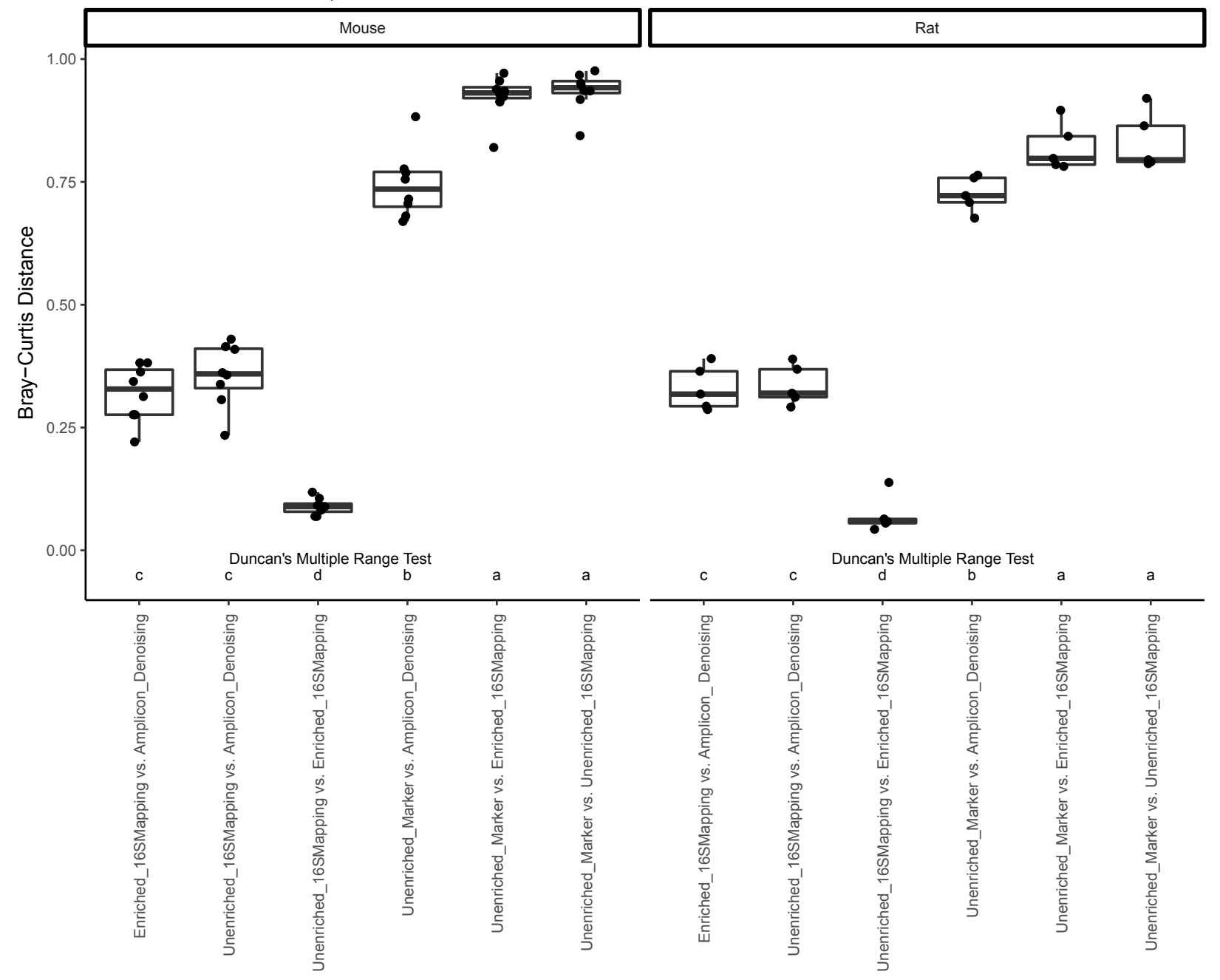

